\title{
Complete monotonicity related to the $k$-polygamma functions with applications
}

\section{Li Yin $^{1 *}$, Jumei Zhang ${ }^{1}$ and XiuLi Lin ${ }^{2}$}

\section{*Correspondence:} yinli_79@163.com

${ }^{1}$ College of Science, Binzhou University, Binzhou, China

Full list of author information is

available at the end of the article

\begin{abstract}
In this paper, we prove complete monotonicity of some functions involving k-polygamma functions. As an application of the main result, we also give new upper and lower bounds of the $k$-digamma function.
\end{abstract}

MSC: Primary 33B15; secondary 26A48

Keywords: Complete monotonicity; k-digamma function; $k$-polygamma functions

\section{Introduction}

The Euler gamma function is defined for all positive real numbers $x$ by

$$
\Gamma(x)=\int_{0}^{\infty} t^{x-1} e^{-t} d t .
$$

The logarithmic derivative of $\Gamma(x)$ is called the psi or digamma function. That is,

$$
\psi(x)=\frac{d}{d x} \ln \Gamma(x)=\frac{\Gamma^{\prime}(x)}{\Gamma(x)}=-\gamma-\frac{1}{x}+\sum_{n=1}^{\infty} \frac{x}{n(n+x)},
$$

where $\gamma=0.5772 \ldots$ is the Euler-Mascheroni constant. The polygamma functions $\psi^{(m)}(x)$ for $m \in \mathbb{N}$ are defined by

$$
\psi^{(m)}(x)=\frac{d^{m}}{d x^{m}} \psi(x)=(-1)^{m} m ! \sum_{n=0}^{\infty} \frac{1}{(n+x)^{m+1}}, \quad x>0 .
$$

The gamma, digamma and polygamma functions play an important role in the theory of special functions, and are closely related to factorial, fractional differential equations, mathematical physics and crops up in many unexpected place in analysis [13-17, 22-28, $40-45]$. For some of the work as regards origin, history, the complete monotonicity, and inequalities of these special functions one may refer to [1-12, 18-21, 29, 30, 33-39] and the references therein.

In 2007, Díaz and Pariguan [16] defined the $k$-analog of the gamma function for $k>0$ and $x>0$ as

$$
\Gamma_{k}(x)=\int_{0}^{\infty} t^{x-1} e^{-\frac{t^{k}}{k}} d t=\lim _{n \rightarrow \infty} \frac{n ! k^{n}(n k)^{\frac{x}{k}-1}}{x(x+k) \cdots(x+(n-1) k)},
$$

(c) The Author(s) 2019. This article is distributed under the terms of the Creative Commons Attribution 4.0 International License (http://creativecommons.org/licenses/by/4.0/), which permits unrestricted use, distribution, and reproduction in any medium, provided you give appropriate credit to the original author(s) and the source, provide a link to the Creative Commons license, and indicate if changes were made. 
where $\lim _{k \rightarrow 1} \Gamma_{k}(x)=\Gamma(x)$. Similarly, we may define the $k$-analog of the digamma and polygamma functions as

$$
\psi_{k}(x)=\frac{d}{d x} \ln \Gamma_{k}(x) \quad \text { and } \quad \psi_{k}^{(m)}(x)=\frac{d^{m}}{d x^{m}} \psi_{k}(x) .
$$

Hence, the authors continued the study of this family of generalized functions, and suggested that many properties of classical gamma, digamma and polygamma functions have a counterpart in this more general setting. It would be natural to generalize the properties of classical functions to the $k$-gamma, digamma and polygamma functions.

It is well known that the $k$-analogues of the digamma and polygamma functions satisfy the following recursive formula and series identities (see $[16,31,32])$ :

$$
\begin{aligned}
\Gamma_{k}(x+k) & =x \Gamma_{k}(x), \quad x>0, \\
\psi_{k}(x) & =\frac{\ln k-\gamma}{k}-\frac{1}{x}+\sum_{n=1}^{\infty} \frac{x}{n k(n k+x)} \\
& =-\int_{0}^{\infty} \frac{e^{-x t}}{1-e^{-k t}} d t,
\end{aligned}
$$

and

$$
\begin{aligned}
\psi_{k}^{(m)}(x) & =(-1)^{m+1} m ! \sum_{n=0}^{\infty} \frac{1}{(n k+x)^{m+1}} \\
& =(-1)^{m+1} \int_{0}^{\infty} \frac{1}{1-e^{-k t}} t^{m} e^{-x t} d t .
\end{aligned}
$$

A function $f$ is said to be completely monotonic on an interval $I$ if $f$ has derivatives of all orders on $I$ and satisfies $(-1)^{n} f^{(n)}(x) \geq 0$ for $x \in I$ and $n \geq 0$. A characterization of completely monotonic functions is given by the Bernstein-Widder theorem which reads that a function $f(x)$ on $x \in[0, \infty)$ is completely monotonic if and only if there exists a bounded and non-decreasing function $g(t)$ such that the integral

$$
f(x)=\int_{0}^{\infty} e^{-x t} d g(t)
$$

converges for $x \in[0, \infty)$. That is, a function $f(x)$ is completely monotonic on $x \in[0, \infty)$ if and only if it is a Laplace transform of a bounded and non-decreasing measure $g(t)$. From the above theorem it follows that completely monotonic functions on $[0, \infty)$ are always strictly completely monotonic unless they are constant (see [34]).

At present, these functions have been extensively studied. In [46], Yin et al. gave a concave theorem and some inequalities for the $k$-digamma function. Furthermore, Yin $e t a l$. [47] showed several monotonic and concave results related to the generalized digamma and polygamma functions. In [48], Zhao, Guo and Qi showed several complete monotonicity of two functions involving the tri- and tetra-gamma functions. Motivated by their work, we give a $k$-analog of their results. Furthermore, we also prove a new double inequality about $k$-polygamma functions. Finally, an application of the main result leads to new upper and lower bounds of the $k$-digamma function. 


\section{Main results}

Lemma 2.1 For $k>0$, we have

$$
\psi_{k}(x)=\frac{\ln k}{k}+\frac{\psi(x / k)}{k}
$$

and

$$
\psi_{k}^{\prime}(x)=\frac{1}{k^{2}} \psi^{\prime}\left(\frac{x}{k}\right) .
$$

Proof Taking logarithms and differentiating on both sides of the formula

$$
\Gamma_{k}(x)=k^{\frac{x}{k}-1} \Gamma\left(\frac{x}{k}\right)
$$

we easily obtain Eq. (2.1). Differentiating on both sides of (2.1), we get (2.2).

Lemma 2.2 For $k>0$, the following recursion formulas hold true:

$$
\begin{aligned}
& \psi_{k}^{\prime}(x+k)=\psi_{k}^{\prime}(x)-\frac{1}{x^{2}}, \\
& \psi_{k}^{\prime \prime}(x+k)=\psi_{k}^{\prime \prime}(x)+\frac{2}{x^{3}} .
\end{aligned}
$$

Proof By using Eq. (1.1), we easily obtain the proof.

Lemma 2.3 ([48, Eq. (12)]) Let $r>0$. Then

$$
\frac{1}{x^{r}}=\frac{1}{\Gamma(r)} \int_{0}^{\infty} t^{r-1} e^{-x t} d t
$$

Theorem 2.1 Let $k>0$. Then the function

$$
f_{k}(x)=(x+k)^{2}\left[\psi_{k}^{\prime}(x)-\frac{1}{x^{2}}-\frac{1}{k(x+k)}\right]
$$

is completely monotonic on $(0, \infty)$.

Proof By the integral representation (1.3) and integration by parts, we have

$$
\begin{aligned}
x \psi_{k}^{\prime}(x) & =x \int_{0}^{\infty} \frac{t e^{-x t}}{1-e^{-k t}} d t=\int_{0}^{\infty} \frac{-t}{1-e^{-k t}} d e^{-x t} \\
& =\left.\frac{-t e^{-x t}}{1-e^{-k t}}\right|_{\infty} ^{0}-\int_{0}^{\infty} \frac{d}{d t}\left(\frac{-t}{1-e^{-k t}}\right) e^{-x t} d t \\
& =\lim _{t \rightarrow 0} \frac{t e^{(k-x) t}}{e^{k t}-1}+\int_{0}^{\infty} \frac{d}{d t}\left(\frac{t e^{k t}}{e^{k t}-1}\right) e^{-x t} d t \\
& =\frac{1}{k}+\int_{0}^{\infty} \frac{e^{2 k t}-e^{k t}-k t e^{k t}}{\left(e^{k t}-1\right)^{2}} e^{-x t} d t .
\end{aligned}
$$


By using (2.6) and integration by parts, we also easily obtain

$$
\begin{aligned}
x^{2} \psi_{k}^{\prime}(x) & =\frac{x}{k}+x \int_{0}^{\infty} \frac{e^{2 k t}-e^{k t}-k t e^{k t}}{\left(e^{k t}-1\right)^{2}} e^{-x t} d t \\
& =\frac{x}{k}+\frac{1}{2}+x \int_{0}^{\infty} \frac{e^{k t}\left(\left(k^{2} t-2 k\right) e^{k t}+2 k+k^{2} t\right)}{\left(e^{k t}-1\right)^{3}} e^{-x t} d t .
\end{aligned}
$$

Furthermore, direct computation results in

$$
f_{k}(x)=x^{2} \psi_{k}^{\prime}(x)+2 k x \psi_{k}^{\prime}(x)+k^{2} \psi_{k}^{\prime}(x)-2-\frac{x}{k}-\frac{2 k}{x}-\frac{k^{2}}{x^{2}} .
$$

Considering (2.6)-(2.8) and Lemma 2.3, we easily get

$$
f_{k}(x)=\frac{1}{2}+\int_{0}^{\infty} \frac{W_{k}(t)}{\left(e^{k t}-1\right)^{3}} e^{-x t} d t
$$

where

$$
W_{k}(t)=\left(k^{2} t-2 k\right) e^{k t}+2 k+k^{2} t
$$

Next, we shall prove $W_{k}(t)>0$ for $t \in(0, \infty)$. Simple calculation gives

$$
W_{k}^{\prime}(t)=k^{3} t e^{k t}-k^{2} e^{k t}+k^{2}
$$

and

$$
W_{k}^{\prime \prime}(t)=k^{4} t e^{k t}>0
$$

From the facts that $W_{k}^{\prime}\left(0^{+}\right)=\lim _{t \rightarrow 0^{+}} W_{k}^{\prime}(t)=0$ and $W_{k}\left(0^{+}\right)=\lim _{t \rightarrow 0^{+}} W_{k}(t)=0$, it follows that the functions $W_{k}^{\prime}(t)$ and $W_{k}(t)$ are increasing and positive on $(0, \infty)$. By computation, we get

$$
f_{k}^{\prime}(x)=\int_{0}^{\infty} \frac{t W_{k}(t)}{\left(e^{k t}-1\right)^{3}} e^{-x t} d t
$$

In consequence, the function $-f_{k}^{\prime}(x)$ is completely monotonic on $(0, \infty)$. This means that

$$
(-1)^{n}\left(-f_{k}^{\prime}(x)\right)^{(n)}=(-1)^{n+1}\left(f_{k}(x)\right)^{(n+1)}>0 .
$$

It is easy to check that $f_{k}(x) \geq \frac{1}{2}>0$. Consequently, the function $f_{k}(x)$ is completely monotonic on $(0, \infty)$.

Corollary 2.1 For $x>0$ and $k>0$, we have

$$
\frac{1}{k(x+k)}+\frac{1}{x^{2}}+\frac{a}{(x+k)^{2}}<\psi_{k}^{\prime}(x)<\frac{1}{k(x+k)}+\frac{1}{x^{2}}+\frac{b}{(x+k)^{2}}
$$

with the best possible constants $a=\frac{1}{2}$ and $b=\frac{\pi^{2}}{6}-1$. 
Proof Complete monotonicity of the function $f_{k}(x)$ implies that the function $f_{k}(x)$ is decreasing on $(0, \infty)$. Therefore, we have

$$
\lim _{x \rightarrow \infty} f_{k}(x)=f_{k}(\infty)<f_{k}(x)<f_{k}\left(0^{+}\right)=\lim _{x \rightarrow 0^{+}} f_{k}(x)
$$

Applying Lemma 2.2, we get

$$
f_{k}(x)=(x+k)^{2}\left[\psi_{k}^{\prime}(x+k)-\frac{1}{(x+k)^{2}}\right] \text {. }
$$

It is easily seen that $f_{k}\left(0^{+}\right)=k^{2} \psi_{k}^{\prime}(k)-1=\frac{\pi^{2}}{6}-1$. On the other hand, using the asymptotic formula (see [1])

$$
\psi^{\prime}(x) \sim \frac{1}{x}+\frac{1}{2 x^{2}}+\frac{1}{6 x^{3}}-\frac{1}{30 x^{5}}+\cdots, \quad x \rightarrow \infty,
$$

and (2.2), we can conclude

$$
f_{k}(x)=\frac{1}{2}+o\left(\frac{1}{(x+k)}\right) \rightarrow \frac{1}{2}, \quad x \rightarrow \infty
$$

This completes the proof.

Remark 2.1 In [47, Lemma 2.4], Yin et al. gave an estimation of $\psi_{k}^{\prime}(x)$ as follows:

$$
\frac{1}{k x}<\psi_{k}^{\prime}(x)<\frac{1}{k x}+\frac{1}{x^{2}}
$$

Here, we give another inequality of $\psi_{k}^{\prime}(x)$.

Theorem 2.2 Let $0<k \leq 1$. Then the functions

$$
\alpha_{k}(x)=k\left(\psi_{k}^{\prime}(x)\right)^{2}+\psi_{k}^{\prime \prime}(x)-\frac{k\left(x^{2}+12 k^{2}\right)}{12 x^{4}(x+k)^{2}}
$$

and

$$
\beta_{k}(x)=\frac{k(x+12 k)}{12 x^{4}(x+k)}-k\left(\psi_{k}^{\prime}(x)\right)^{2}-\psi_{k}^{\prime \prime}(x)
$$

are completely monotonic on $(0, \infty)$. As a direct result, for $0<k \leq 1$ and $x \in(0, \infty)$, we have the following double inequality:

$$
\frac{k\left(x^{2}+12 k^{2}\right)}{12 x^{4}(x+k)^{2}}<k\left(\psi_{k}^{\prime}(x)\right)^{2}+\psi_{k}^{\prime \prime}(x)<\frac{k(x+12 k)}{12 x^{4}(x+k)} .
$$

Proof By the recursion formula (2.4), we get

$$
\begin{aligned}
\alpha_{k}(x)-\alpha_{k}(x+k)= & k\left[\psi_{k}^{\prime}(x)+\psi_{k}^{\prime}(x+k)\right]\left[\psi_{k}^{\prime}(x)-\psi_{k}^{\prime}(x+k)\right] \\
& +\psi_{k}^{\prime \prime}(x)-\psi_{k}^{\prime \prime}(x+k)-\left[\frac{k\left(x^{2}+12 k^{2}\right)}{12 x^{4}(x+k)^{2}}-\frac{k\left((x+k)^{2}+12 k^{2}\right)}{12(x+k)^{4}(x+2 k)^{2}}\right]
\end{aligned}
$$




$$
\begin{aligned}
& =\frac{2 k}{x^{2}}\left[\psi_{k}^{\prime}(x)-\frac{1}{2 x^{2}}-\frac{1}{k x}-\frac{\left(x^{2}+12 k^{2}\right)}{24 x^{2}(x+k)^{2}}+\frac{x^{2}\left((x+k)^{2}+12 k^{2}\right)}{24(x+k)^{4}(x+2 k)^{2}}\right] \\
& =\frac{2 k}{x^{2}} g_{k}(x),
\end{aligned}
$$

where

$$
\begin{aligned}
g_{k}(x)= & \psi_{k}^{\prime}(x)-\frac{1}{x^{2}}-\frac{k^{2}}{2(x+k)^{4}}-\frac{2 k}{(x+k)^{3}} \\
& +\frac{7}{2(x+k)^{2}}-\frac{43}{6 k(x+k)}+\frac{37}{6 k(x+2 k)}+\frac{13}{6(x+2 k)^{2}} .
\end{aligned}
$$

Applying (1.3) and (2.5), we have

$$
g_{k}(x)=\frac{1}{12 k} \int_{0}^{\infty} \frac{q_{k}(x)}{e^{k t}-1} e^{-(x+2 k) t} d t,
$$

where

$$
\begin{aligned}
q_{k}(x)= & e^{2 k t}\left(k^{3} t^{3}-12 k^{2} t^{2}+12 k t+42 t-86\right) \\
& +e^{k t}\left(-k^{3} t^{3}+12 k^{2} t^{2}-16 t+160\right)-26 t-74
\end{aligned}
$$

Direct calculation yields

$$
\begin{aligned}
q_{k}^{\prime}(x)= & e^{2 k t}\left(2 k^{4} t^{3}-21 k^{3} t^{2}+84 k t+42-160 k\right) \\
& +e^{k t}\left(-k^{4} t^{3}+9 k^{3} t^{2}+\left(24 k^{2}-16 k\right) t+160 k-16\right)-26
\end{aligned}
$$

and

$$
q_{k}^{\prime \prime}(x)=e^{k t} \lambda_{k}(x),
$$

where

$$
\begin{aligned}
\lambda_{k}(x)= & e^{k t}\left(4 k^{5} t^{3}-36 k^{4} t^{2}+168 k^{2} t-42 k^{3} t+168 k-320 k^{2}\right) \\
& -k^{5} t^{3}+6 k^{4} t^{2}+\left(42 k^{3}-16 k^{2}\right) t+184 k^{2}-32 k .
\end{aligned}
$$

Further computation gives

$$
\begin{aligned}
\lambda_{k}^{\prime}(x)= & k^{2}\left(-16+42 k+12 k^{2} t-3 k^{3} t^{2}\right) \\
& +2 k^{2} e^{k t}\left(168-57 k^{2} t-12 k^{3} t^{2}+2 k^{4} t^{3}-181 k+84 k t\right), \\
\lambda_{k}^{\prime \prime}(x)= & 2 k^{3}\left[-3 k(-2+k t)+e^{k t}\left(252-81 k^{2} t-6 k^{3} t^{2}+2 k^{4} t^{3}+14 k(-17+6 t)\right)\right], \\
\lambda_{k}^{\prime \prime \prime}(x)= & -6 k^{5}+2 k^{4} e^{k t}\left[336-319 k+\left(84 k-93 k^{2}\right) t+2 k^{4} t^{3}\right] .
\end{aligned}
$$

Since $0<k \leq 1$, the function $336-319 k+\left(84 k-93 k^{2}\right) t+2 k^{4} t^{3}$ attains minimum value $14-3 \sqrt{6}$ as $t \rightarrow \sqrt{3 / 2}$ and $k \rightarrow 1$. This implies $\lambda_{k}^{\prime \prime \prime}(x)>0$. From the facts $\lambda_{k}^{\prime}(0)=k^{2}(320-$ $320 k)>0$ and $\lambda_{k}^{\prime \prime}(0)=2 k^{3}(252-232 k)>0$, it follows that the functions $\lambda_{k}^{\prime}(x), \lambda_{k}^{\prime \prime}(x), \lambda_{k}^{\prime \prime \prime}(x)$ 
are increasing and positive on $(0, \infty)$. Thus, the derivative $q_{k}^{\prime \prime}(x)$ is positive, and so the function $q_{k}^{\prime}(x)$ is increasing on $(0, \infty)$. Since $q_{k}^{\prime}(0)=0$, the function $q_{k}^{\prime}(t)$ is positive and $q_{k}(t)$ is increasing on $(0, \infty)$. Since $q_{k}(0)=0, q_{k}(t)$ is positive on $(0, \infty)$.

Positivity of $q_{k}(t)$ leads to the complete monotonicity of $g_{k}(x)$ on $(0, \infty)$. Since $\frac{2 k}{x^{2}}$ is completely monotonic on $(0, \infty)$ and the product of finite completely monotonic functions is also completely monotonic, the difference $\alpha_{k}(x)-\alpha_{k}(x+k)$ is completely monotonic on $(0, \infty)$. That is,

$$
(-1)^{n}\left(\alpha_{k}(x)-\alpha_{k}(x+k)\right)^{(n)}=(-1)^{n}\left(\alpha_{k}(x)\right)^{(n)}-(-1)^{n}\left(\alpha_{k}(x+k)\right)^{(n)}>0 .
$$

By mathematical induction, we get

$$
(-1)^{n}\left(\alpha_{k}(x)\right)^{(n)}>(-1)^{n}\left(\alpha_{k}(x+k)\right)^{(n)}>\cdots>(-1)^{n}\left(\alpha_{k}(x+i k)\right)^{(n)} \rightarrow 0 .
$$

So, we prove that the function $\alpha_{k}(x)$ is completely monotonic on $(0, \infty)$. A completely similar method may apply to the function $\beta_{k}(x)$. Here, we omit the details for the sake of simplicity.

Remark 2.2 Taking $k=1$ in inequality (2.9), we obtain [48, Theorem 1(8)].

\section{An application}

In this section, we shall give an application to obtain the bounds of the $k$-digamma function by using Theorem 2.2.

Lemma 3.1 For $x>0$ and $0<k \leq 1$, we have $\psi_{k}^{\prime}(x) e^{k \psi_{k}(x)}<\frac{1}{k}$.

Proof By using inequality (2.9), we have

$$
\frac{d}{d x}\left(k \psi_{k}(x)+\ln \psi_{k}^{\prime}(x)\right)>0, \quad x>0 .
$$

This means that $k \psi_{k}(x)+\ln \psi_{k}^{\prime}(x)$ is strictly increasing on $(0, \infty)$. By [31] for $x>0$ and $0<k \leq 1$, we have

$$
\frac{1}{k} \ln x-\frac{1}{x}<\psi_{k}(x)<\frac{1}{k} \ln x .
$$

This gives

$$
x \psi_{k}^{\prime}(x) e^{-\frac{k}{x}}<\psi_{k}^{\prime}(x) e^{k \psi_{k}(x)}<x \psi_{k}^{\prime}(x)
$$

Using Eq. (2.2) and an asymptotic representation of $\psi(x)$, we can get

$$
\psi_{k}^{\prime}(x) \sim \frac{1}{k x}+\frac{1}{2 x^{2}}+\frac{k}{6 x^{3}}-\cdots, \quad x \rightarrow \infty .
$$

Furthermore, we get $\lim _{x \rightarrow \infty} x \psi_{k}^{\prime}(x)=\frac{1}{k}$. Hence, by inequality (3.1), we find that $\lim _{x \rightarrow \infty} k \psi_{k}(x)+\ln \psi_{k}^{\prime}(x)=\ln \left(\frac{1}{k}\right)$. So the proof follows from the monotonicity of the function $k \psi_{k}(x)+\ln \psi_{k}^{\prime}(x)$. 
Lemma 3.2 Let $0<k \leq 1$. Then the function $A_{k}(x)=\frac{1}{k} e^{k \psi_{k}(x+k)}-\frac{x}{k}$ is strictly decreasing and strictly convex on $(-k, \infty)$.

Proof Simple computation yields

$$
A_{k}^{\prime}(x)=\psi_{k}^{\prime}(x+k) e^{k \psi_{k}(x+k)}-\frac{1}{k}
$$

and

$$
A_{k}^{\prime \prime}(x)=\left[k\left(\psi_{k}^{\prime}(x+k)\right)^{2}+\psi_{k}^{\prime \prime}(x+k)\right] e^{k \psi_{k}(x+k)} .
$$

By applying Lemma (3.1) and inequality (2.9), we easily obtain $A_{k}^{\prime}(x)<0$ and $A_{k}^{\prime \prime}(x)>0$. The proof is complete.

Theorem 3.1 For $0<k \leq 1$ and $x>0$, we have

$$
\frac{\ln k}{k}+\frac{1}{k} \ln \left(\frac{x}{k}+\frac{1}{2}\right)-\frac{1}{x}<\psi_{k}(x)<\frac{\ln k}{k}+\frac{1}{k} \ln \left(\frac{x}{k}+e^{-\gamma}\right)-\frac{1}{x} .
$$

The constants $\frac{1}{2}$ and $e^{-\gamma}$ in (3.2) are the best possible as $x \rightarrow \infty$.

Proof Direct calculation results in $\lim _{x \rightarrow 0^{+}} A_{k}(x)=e^{k\left[\psi_{k}(k)-\frac{\ln k}{k}\right]}=e^{-\gamma}$ and $\lim _{x \rightarrow \infty} A_{k}(x)=$ $\frac{1}{2}$. Noting that the function $A_{k}(x)$ is strictly increasing on $(0, \infty)$, we easily complete the proof.

\section{Conclusion}

In this paper, we mainly proved the following theorems: Let $k>0$. Then the function

$$
f_{k}(x)=(x+k)^{2}\left[\psi_{k}^{\prime}(x)-\frac{1}{x^{2}}-\frac{1}{k(x+k)}\right]
$$

is completely monotonic on $(0, \infty)$.

Let $0<k \leq 1$. Then the functions

$$
\alpha_{k}(x)=k\left(\psi_{k}^{\prime}(x)\right)^{2}+\psi_{k}^{\prime \prime}(x)-\frac{k\left(x^{2}+12 k^{2}\right)}{12 x^{4}(x+k)^{2}}
$$

and

$$
\beta_{k}(x)=\frac{k(x+12 k)}{12 x^{4}(x+k)}-k\left(\psi_{k}^{\prime}(x)\right)^{2}-\psi_{k}^{\prime \prime}(x)
$$

are completely monotonic on $(0, \infty)$. As an application of Theorem 2.2, we also give new upper and lower bounds of the $k$-digamma function. 


\section{Funding}

This work was supported by National Natural Science Foundation of China (Grant No. 11701320 and 11601036), the Science and Technology Foundations of Shandong Province (Grant No. J17KA161 and J18KB105) and Science Foundation of Binzhou University (Grant No. BZXYL1704).

\section{Competing interests}

The authors declare that they have no competing interests.

\section{Authors' contributions}

All authors contributed equally to the manuscript and read and approved the final manuscript.

\section{Author details}

${ }^{1}$ College of Science, Binzhou University, Binzhou, China. ${ }^{2}$ College of Mathematics Science, Qufu Normal University, Qufu, China.

\section{Publisher's Note}

Springer Nature remains neutral with regard to jurisdictional claims in published maps and institutional affiliations.

Received: 8 May 2019 Accepted: 16 August 2019 Published online: 28 August 2019

\section{References}

1. Abramowitz, M., Stegun, I. (eds.): Handbook of Mathematical Functions with Formulas, Graphs and Mathematical Tables. National Bureau of Standards, Dover, New York (1965)

2. Agarwal, P.: Some inequalities involving Hadamard type k-fractional integral operators. Math. Methods Appl. Sci. 40(11), 3832-3891 (2017)

3. Agarwal, P., Dragomir, S.S., Jleli, M., Samet, B.: Advances in Mathematical Inequalities and Applications. Birkhäuser, Basel (2018)

4. Agarwal, P., Jleli, M., Samet, B.: Fixed Point Theory in Metric Spaces. Springer, Singapore (2018)

5. Agarwal, P., Jleli, M., Tomar, M.: Certain Hermite-Hadamard type inequalities via generalized $k$-fractional integrals. J. Inequal. Appl. 2017, 55 (2017)

6. Agarwal, P., Rogosin, S.V., Karimov, E.T.: Certain fractional integral operators and the generalized incomplete hypergeometric functions. Appl. Appl. Math. 8(2), 333-345 (2013)

7. Agarwal, R.P., Luo, M.J., Agarwal, P.: On the extended Appell-Lauricella hypergeometric functions and their applications. Filomat 31, 12 (2017)

8. Alzer, H.: On some inequalities for the gamma and psi function. Math. Comput. 66, 373-389 (1997)

9. Alzer, H.: Sharp inequalities for the digamma and polygamma functions. Forum Math. 16, 181-221 (2004)

10. Alzer, H.: Sharp inequalities for the harmonic numbers. Expo. Math. 24(4), 385-388 (2006)

11. Batir, N.: Some new inequalities for gamma and polygamma functions. J. Inequal. Pure Appl. Math. 6(4), Article ID 103 (2005)

12. Batir, N.: On some properties of digamma and polygamma functions. J. Math. Anal. Appl. 328(1), 452-465 (2014)

13. Chiu, S.N., Yin, C.-C.: On the complete monotonicity of the compound geometric convolution with applications to risk theory. Scand. Actuar. J. 2(2014), 116-124 (2014)

14. Choi, J.S., Agarwal, P.: Some new Saigo type fractional integral inequalities and their analogues. Abstr. Appl. Anal. 2014, 11 (2014)

15. Coffey, M.C.: On one dimensional digamma and polygamma series related to the evaluation of Feynman diagrams. J. Comput. Appl. Math. 183, 84-100 (2005)

16. Díaz, R., Pariguan, E.: On hypergeometric functions and Pochhammer k-symbol. Divulg. Mat. 15(2), 179-192 (2007)

17. Dong, H., Yin, C.-C.: Complete monotonicity of the probability of ruin and De Finetti's dividend problem. J. Syst. Sci. Complex. 25(1), 178-185 (2012)

18. Guo, B.-N., Qi, F.: Some properties of the psi and polygamma functions. Hacet. J. Math. Stat. 39(2), 219-231 (2010)

19. Guo, B.-N., Qi, F.: Two new proofs of the complete monotonicity of a function involving the psi function. Bull. Korean Math. Soc. 47(1), 103-111(2010)

20. Guo, B.-N., Qi, F., Srivastava, H.M.: Some uniqueness results for the non-trivially complete monotonicity of a class of functions involving the polygamma and related functions. Integral Transforms Spec. Funct. 21(11), 849-858 (2010)

21. Guo, B.-N., Zhao, J.-L., Qi, F.: A completely monotonic function involving divided differences of the tri- and tetra-gamma functions. Math. Slovaca 63(3), 469-478 (2013)

22. Guo, Y.-X.: Solvability for a nonlinear fractional differential equation. Bull. Aust. Math. Soc. 80, 125-138 (2009)

23. Jain, S., Mehrez, K., Baleanu, D., Agarwal, P.: Certain Hermite-Hadamard inequalities for logarithmically convex functions with applications. Mathematics 7(2), 163 (2019)

24. Krasniqi, F., Shabani, A.S.: Convexity properties and inequalities for a generalized gamma functions. Appl. Math. E-Notes 10, 27-35 (2010)

25. Krasniqi, V., Mansour, T., Shabani, A.S.: Some monotonicity properties and inequalities for $\Gamma$ and $\zeta$ functions. Math. Commun. 15(2), 365-376 (2010)

26. Krasniqi, V., Mansour, T., Shabani, A.S.: Some inequalities for $q$-polygamma function and $\zeta_{q}$-Riemann zeta functions. Ann. Math. Inform. 37, 95-100 (2010)

27. Lin, X.-L., Zhao, Z.-Q.: Iterative technique for a third-order differential equation with three-point nonlinear boundary value conditions. Electron. J. Qual. Theory Differ. Equ. 2016, 12 (2016)

28. Liu, X.-H., Zhang, L.-H., Agarwal, P., Wang, G.-T.: On some new integral inequalities of Gronwall-Bellman-Bihari type with delay for discontinuous functions and their applications. Indag. Math. 27(1), 1-10 (2016)

29. Merkle, M.: Inequalities for the gamma function via convexity. In: Cerone, P., Dragomir, S.S. (eds.) Advances in Inequalities for Special Functions, pp. 81-100. Nova Science Publishers, New York (2008) 
30. Nantomah, K.: Convexity properties and inequalities concerning the $(p, k)$-gamma functions. Commun. Fac. Sci. Univ. Ank. Sér. A1 Math. Stat. 66(2), 130-140 (2017)

31. Nantomah, K., Merovci, F., Nasiru, S.: Some monotonic properties and inequalities for the $(p, q)$-gamma function. Kragujev. J. Math. 42(2), 287-297 (2018)

32. Nantomah, K., Prempeh, E., Twum, S.B.: On a $(p, k)$-analogue of the gamma function and some associated inequalities. Moroccan J. Pure Appl. Anal. 2(2), 79-90 (2016)

33. Qi, F.: Bounds for the ratio of two gamma functions. J. Inequal. Appl. 2010, 1 (2010)

34. Qi, F., Chen, C.-P.: Some completely monotonic and polygamma functions. J. Aust. Math. Soc. 80, 81-88 (2006)

35. Qi, F., Cui, R.-Q., Chen, C.-P., Guo, B.-N.: Some completely monotonic functions involving polygamma functions and an application. J. Math. Anal. Appl. 310(1), 303-308 (2005)

36. Qi, F., Guo, B.-N.: Completely monotonic functions involving divided differences of the di- and tri-gamma functions and some applications. Commun. Pure Appl. Anal. 8(6), 1975-1989 (2009)

37. Qi, F., Guo, B.-N.: Necessary and sufficient conditions for functions involving the tri- and tetra-gamma functions to be completely monotonic. Adv. Appl. Math. 44(1), 71-83 (2010)

38. Qi, F., Guo, B.-N.: A class of completely monotonic functions involving divided differences of the psi and tri-gamma functions and some applications. J. Korean Math. Soc. 48(3), 655-667 (2011)

39. Qi, F., Guo, S.-L., Guo, B.-N.: Completely monotonicity of some functions involving polygamma functions. J. Comput. Appl. Math. 233, 2149-2160 (2010)

40. Ruzhansky, M., Je, C.Y., Agarwal, P., Area, I.: Advances in Real and Complex Analysis with Applications. Springer, Singapore (2018)

41. Saad, K., Samuel, O., Iyiola, S., Agarwal, P.: An effective homotopy analysis method to solve the cubic isothermal auto-catalytic chemical system. AIMS Math. 3(1), 183-194 (2018)

42. Tariboon, J., Ntouyas, S.K., Agarwal, P.: New concepts of fractional quantum calculus and applications to impulsive fractional q-difference equations. Adv. Differ. Equ. 2015(1),18 (2015)

43. Wang, Y., Liu, L.-S., Wu, Y.-H.: Existence and uniqueness of a positive solution to singular fractional differential equations. Bound. Value Probl. 2012, 81 (2012)

44. Wang, Y., Liu, L.-S., Wu, Y.-H.: Positive solutions for a class of higher-order singular semipositone fractional differential system. Adv. Differ. Equ. 2014, 268 (2014)

45. Xu, R., Meng, F.-W.: Some new weakly singular integral inequalities and their applications to fractional differential equations. J. Inequal. Appl. 2016, 78 (2016)

46. Yin, L., Huang, L.-G., Lin, X.-L., Wang, Y.-L.: Monotonicity, concavity, and inequalities related to the generalized digamma function. Adv. Differ. Equ. 2018, 246 (2018)

47. Yin, L., Huang, L.-G., Song, Z.-M., Dou, X.-K.: Some monotonicity properties and inequalities for the generalized digamma and polygamma functions. J. Inequal. Appl. 2018, 249 (2018)

48. Zhao, J.-L., Guo, B.-N., Qi, F.: Complete monotonicity of two functions involving the tri- and tetra-gamma functions. Period. Math. Hung. 65(1), 147-155 (2012)

\section{Submit your manuscript to a SpringerOpen ${ }^{\circ}$ journal and benefit from:}

- Convenient online submission

- Rigorous peer review

- Open access: articles freely available online

- High visibility within the field

- Retaining the copyright to your article

Submit your next manuscript at $\gg$ springeropen.com 